\title{
Esports and Domain General Research for High Performance Teams
}

\author{
Jason G. Reitman \\ jreitman@uci.edu \\ University of California, Irvine \\ Irvine, California, USA
}

\author{
Constance Steinkuehler \\ const@uci.edu \\ University of California, Irvine \\ Irvine, California, USA
}

\begin{abstract}
Through our work studying team communication and awareness under stress in elite and developing esports teams, we are building a foundation for testing the generalizability of findings between similar teams in disparate domains. Team taxonomy literature implies that findings from one domain might have implications for a team with similar characteristics in another domain. Researchers have even demonstrated that communication strategies and leadership structures can successfully transfer across fields, yet it is rare for studies that attempt to apply findings across seemingly disparate teams to discuss their results in terms of existing taxonomies. That disconnect between theoretical taxonomies and practical interventions means that even studies that successfully transfer team practices across domains are framed as case studies without generalizable implications. Here we argue for the study of esports teams to work toward generalizability with high performance teams more broadly, and we describe current work with that goal.
\end{abstract}

\section{CCS CONCEPTS}

- Human-centered computing $\rightarrow$ Computer supported cooperative work.

\section{KEYWORDS}

teams, esports, taxonomy, communication

ACM Reference Format:

Jason G. Reitman and Constance Steinkuehler. 2021. Esports and Domain General Research for High Performance Teams. In Proceedings of ACM Conference (CHI'21). ACM, New York, NY, USA, 4 pages.

\section{INTRODUCTION}

Teams research has produced a number of team taxonomies, like those described by Wildman et al. [15], which detail characteristics and task types to establish categories of teams that transcend the fields in which they work. In parallel with those theoretical efforts, intervention studies and experimental designs have successfully translated findings across domains, like Catchpole et al.'s [2] work decreasing errors during patient handover between Operating Room and Intensive Care Unit teams using data from Formula 1

Permission to make digital or hard copies of all or part of this work for personal or classroom use is granted without fee provided that copies are not made or distributed for profit or commercial advantage and that copies bear this notice and the full citation on the first page. Copyrights for components of this work owned by others than ACM must be honored. Abstracting with credit is permitted. To copy otherwise, or republish, to post on servers or to redistribute to lists, requires prior specific permission and/or a fee. Request permissions from permissions@acm.org.

CHI'21, May 2021, Online

(C) 2021 Association for Computing Machinery.

ACM ISBN 978-x-xxxx-xxxx-x/YY/MM...\$15.00 pit crews and aircraft navigation. It is rare, however, for studies that attempt to generalize team practices across domains to discuss their findings in terms of taxonomic theory.

Esports teams share key characteristics with the kind of team in Catchpole et al.'s [2] study. This paper discusses those characteristics, this category of team, and the implications for research of esports teams more broadly. We suggest that better understanding the current disconnect between team taxonomies and cross-domain team interventions, in addition to using esports as a context to study a particular kind of team, will provide opportunities for researchers to generalize findings from esports teams to similar teams in higher consequence environments.

\section{CHARACTERISTICS OF ESPORTS AND ISOMORPHIC TEAMS}

Table 1 uses examples from teamwork in League of Legends (LoL) and three non-esports contexts to illustrate how the characteristics that define this category manifest across domains. These characteristics come from teams research literature in CSCW (e.g. [6]), cognitive science (e.g. [9]), and management (e.g. [8]) and from the authors' previous and current work studying elite esports teams [12] [13] [16]. The alignment illustrated by this table suggests that there is a category of team whose members work under temporal pressure, require attentional synchrony to accomplish tasks, require some communication through technology as opposed to face to face, have common access to at least some task-relevant information, share common goals, work in clearly differentiated roles, and employ an intentional leadership structure. This definition intentionally omits any reference to the content of the teams' goals and tasks. The strength of this definition is that omission, since it allows comparisons of teams that work with similar structures and under similar pressures but may not work in similar domains.

LoL teams, for example, must perform under time pressure both because they are racing to destroy their opponent's base before their opponent destroys theirs and because success in teamfights depends on split-second coordination of multiple players using specific abilities to engage or disengage enemy players. That level of coordination in teamfights also requires attentional synchrony. In order to understand when and how they need to act to land combinations of attacks with their teammates, each player attends to the same stimuli. An example is Blitzcrank's hook ability. If Blitzcrank hits an enemy with its hook, the target is pulled toward Blitzcrank, where they are vulnerable to further attacks. Every time Blitzcrank uses its hook, though, it cannot be used for a few seconds ${ }^{1}$. When deciding if and when to engage against a team

${ }^{1}$ Base cooldown of 20 seconds at match start. By mid- to late-game, when team fights are more common, it can have a base cooldown of 16 seconds minus $40 \%$ to $45 \%$ 
with a Blitzcrank, success against a skilled opponent requires each player attend to that hook simultaneously. The team's best chance of winning the fight is immediately after hook is used, because they have at least 8.8 seconds where they can engage without the threat of being pulled toward Blitzcrank. If some team members are not attending to the hook, valuable time is wasted alerting them that Blitzcrank just used the ability. Instead of immediately attacking, the team either waits and the opportunity is lost, or some members attack and some do not, splitting the team and leaving them vulnerable. Blitzcrank's hook is just one example of an ability that has to be played around in order to win teamfights. In any given game, each team has a number of such tools to work with and against. Communication about teamfight strategy must therefore happen in large part before it is actually time to fight, because once a fight begins, communication is often reduced to single-word or ping commands to announce targets or moments to act. Without each player attending to the same stimuli, those commands lose context and acting upon them at the right time in the right manner is not consistently feasible.

Emergency department staff do not have a competitor. Their goals have nothing to do with out-smarting or out-coordinating another team. The tasks they complete to achieve their goals look very different from fighting in competitive video games, changing the flap orientation of airplane wings, or swapping tires on a Formula 1 car. The structures and pressures within which they work, however, are similar enough to a Formula 1 pit-stop and an aircraft landing procedure for Catchpole et al. [2] to design a new patient handover protocol based on observations of pit-stops and interviews with a race director and two aircraft captains. The new protocol reduced errors in task completion and omissions of information transferred during patient handover from the operating theater to the intensive care unit (ICU), in addition to shortening the duration of that process. From those observations and interviews, the researchers made changes to the leadership structure, temporal ordering of tasks, task allocation, safety checks, and communication structure of the teams of doctors and nurses that handle the transfer of a patient from surgery to ICU. Each of these changes made part of the process more explicitly structured. For instance, based on the clear responsibility and leadership of the lollipop man's role in a pit-stop and the captain's role on a flight, the new protocol gave clear "overall responsibility for coordinating the team" to the anesthetist for the duration of the handover and to the intensivist at the end of the handover, where previously it was "unclear who was in charge" at any given moment [2]. Similarly, the expectations of the structure of communication were made explicit based on the "very little verbal communication during a pit-stop" and the "explicit communication strategies used to ensure a calm and organized atmosphere" [2] in an aircraft. During patient handover, the researchers limited speaking to the anesthetist and surgeon before allowing general discussion, instead of the "several simultaneous discussions" that occurred under the old, less explicitly structured, handover protocol. Every measure showed statistically significant improvement under the new protocol developed by adapting successful practices used by teams from other fields.

maximum cooldown reduction from items, leaving a minimum of 8.8 seconds between casts. This is considered a long cooldown for an ability, generally only surpassed by ultimate abilities and summoner spells.
Among emergency department staff in hospitals, temporal pressure is the result of emergency rooms full of sick and injured patients waiting to be cared for and, in some cases, government mandates of the maximum amount of time a hospital is permitted before tending to a new patient waiting in the emergency room [4]. For Formula 1 pit crews it comes from their team's foremost goal: be faster than everyone else without making errors. In a cockpit, temporal pressure arises from the need to decide how to alter flight to reach a safe speed, heading, and altitude for landing [9]. Catchpole et al.'s [2] results give credence to this idea that teams can learn from practices used in disparate domains. The goal of establishing this category of isomorphic teams around characteristics found in LoL teams is to allow lessons learned from researching esports teams to be useful in other contexts where data are harder to collect.

Note that Table 1 is not itself a taxonomy. Rather it outlines one category of isomorphic teams that would be described within a taxonomy. While that taxonomy will be helpful for understanding the range of teams across domains that might learn from each other, detailing this one category is enough to begin work that might generalize between esports and isomorphic teams.

\section{EMPIRICAL WORK TOWARD GENERALIZATION}

Better understanding how teams communicate under stress could have implications for team training and safety beyond the world of competitive video games. Looking at how communication relates to performance is not new. Researchers have highlighted how particular communication practices, like checklists, make aircraft navigation [9] and patient care safer and more efficient [2]. Catchpole et al. even translated Formula 1 pit crew communication structures to improve information transfer between surgery and ICU teams during patient handoff [2]. In doing so, they reduced technical errors and information handover omissions, all while shortening the duration of handovers. Teams in disparate domains, then, can benefit from similar communication practices under similar pressures.

With a $2 \times 2$ factorial design, we are currently exploring relationships between experience - both in gameplay and in teamwork and aspects of team communication that apply to awareness, coordination, and in-game mentoring. Using game telemetry and biometric data to identify stressful periods of play, we analyze team communication content, style, and structure and how they relate to teams' in-game behavior. This expert-novice comparison will help uncover what characteristics of team communication are displayed by players and teams at different levels of competitive performance. How a team communicates amid a stressful event may well determine that team's performance or even their ability to learn from experience, regardless of domain. If esports provide a context for examining teams more broadly, understanding the differences in communication practices between expert and novice LoL teams can help us develop and test similar practices in teams under similar pressures.

This work focuses on communication in LoL, in particular, because of similarities between competitive LoL teams and literature describing small teams in domains outside of esports, like emergency medicine and air traffic control. If expert LoL communication practices relate to stress responses that do not hinder performance, 
Table 1: Characteristics and examples from League of Legends and isomorphic teams.

\begin{tabular}{|c|c|c|c|c|}
\hline Feature & Esports (LoL) & Emergency medicine & Aircraft navigation & Formula 1 \\
\hline Temporal pressure & $\begin{array}{l}\text { Split-second } \\
\text { coordination [12] }\end{array}$ & $\begin{array}{l}\text { "The four-hour target" [4] } \\
\text { "The handover of patients } \\
\text { from theater to ICU..." [2] }\end{array}$ & $\begin{array}{l}\text { Precise timing of } \\
\text { changes in elevation, } \\
\text { heading, and speed } \\
\text { to stay safe and } \\
\text { on schedule [9] }\end{array}$ & $\begin{array}{l}\text { "The pit-stop... (change } \\
\text { four tyres and fill with fuel) } \\
\text { under huge time pressure } \\
\text { (approx } 7 \mathrm{~s} \text { ) with minimal } \\
\text { error" [2] }\end{array}$ \\
\hline Attentional synchrony & $\begin{array}{l}\text { Target acquisition in } \\
\text { teamfights } \\
\text { Minion wave } \\
\text { management }\end{array}$ & $\begin{array}{l}\text { Clinicians look at patient } \\
\text { records together when } \\
\text { problem-solving [1] }\end{array}$ & $\begin{array}{l}\text { Pilots' and copilots' } \\
\text { "redundancy in the } \\
\text { visual field" [5] } \\
\text { "Crew coordination } \\
\text { cross-checking } \\
\text { procedures" [9] }\end{array}$ & $\begin{array}{l}\text { Sensor data and warnings } \\
\text { displayed simultaneously } \\
\text { to driver, technical team, } \\
\text { and race engineer }\end{array}$ \\
\hline Common access to information & $\begin{array}{l}\text { On all team members' } \\
\text { screens: } \\
\text { - Minimap } \\
\text { - Teammate health } \\
\text { and mana } \\
\text { - Teammate and } \\
\text { enemy items } \\
\text { - Text chat log }\end{array}$ & $\begin{array}{l}\text { "Shared knowledge of } \\
\text { task structure and team } \\
\text { processes" [14] }\end{array}$ & $\begin{array}{l}\text { Pilots and copilots have } \\
\text { identical sets of } \\
\text { gauges, and checklist } \\
\text { procedure sometimes } \\
\text { necessitate joint } \\
\text { attention to a specific } \\
\text { gauge [9] }\end{array}$ & $\begin{array}{l}\text { Sensor data avilable to } \\
\text { driver, race engineer, and } \\
\text { technical team }\end{array}$ \\
\hline Common goals & $\begin{array}{l}\text { Destroy enemy nexus } \\
\text { - Destroy towers } \\
\text { - Control objectives } \\
\text { - Control vision } \\
\text { - Amass gold and } \\
\text { experience }\end{array}$ & $\begin{array}{l}\text { "Well-articulated and } \\
\text { understood goals and } \\
\text { vision are necessary for } \\
\text { giving ... as well as } \\
\text { integrating feedback..." } \\
{[14]}\end{array}$ & 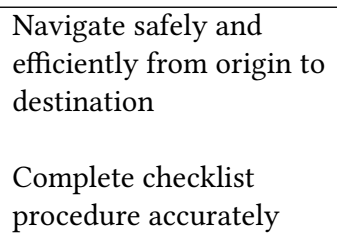 & $\begin{array}{l}\text { Win race } \\
\text { - Complete laps and pit } \\
\text { stops faster than } \\
\text { opponents } \\
\text { - Execute race strategy }\end{array}$ \\
\hline Differentiated roles & $\begin{array}{l}\text { Map positions } \\
\text { (e.g. top laner, } \\
\text { jungler) } \\
\text { Teamfight roles } \\
\text { (e.g. tank, engage) }\end{array}$ & $\begin{array}{l}\text { "Allows teams member to } \\
\text { formulate accurate } \\
\text { expectations of ... needs } \\
\text { during high-stress work } \\
\text { episodes." [14] }\end{array}$ & $\begin{array}{l}\text { "Explicit acknowledged } \\
\text { allocation of tasks for } \\
\text { emergencies" [2] }\end{array}$ & $\begin{array}{l}\text { "Each team member has } \\
\text { only one or two clearly } \\
\text { defined tasks." [2] }\end{array}$ \\
\hline Intentional leadership structure & Shotcaller & $\begin{array}{l}\text { Resuscitation team } \\
\text { leaders [3] }\end{array}$ & Captain [2] & $\begin{array}{l}\text { - Race team: race } \\
\text { engineer } \\
\text { - Technical team: chief } \\
\text { technical officer } \\
\text { - Pit crew: lollipop man [2] }\end{array}$ \\
\hline
\end{tabular}

the content, style, and structure of that communication might also benefit teams in contexts with more severe consequences. If expert LoL communication practices do not relate to consistent performance under pressure, there could be an opportunity to adapt communication practices of other domains to test against existing practices in esports. In other words, this is an approach to esports research that would inform life outside of competitive gaming. As
McCarthy argued for "chess as the Drosophila of artificial intelligence" [10], this work argues for esports as a Drosophila of high performance team research.

\section{DISCUSSION}

In 1973, Newell called on cognitive science and artificial intelligence research 
to focus a series of experimental and theoretical studies around a single complex task, the aim being to demonstrate that one has a sufficient theory of a genuine slab of human behavior. All of the studies would be designed to fit together and add up to a total picture in detail. [11]

In 2017, Gray asserted that "games represent the type of experimental paradigm that Newell was advocating and ... provide the technologies and data needed to realize his vision" [7]. Taken together, the theoretical and methodological suggestions discussed in this article agree that elite esports play is such a setting.

While our current work focuses on communication under pressure in LoL teams, esports teams from different games can display distinct characteristics. In other words, while LoL teams may belong to the category of team described by Table 1, teams from other esports may share characteristics with other kinds of teams. Delineating the team characteristics necessitated by different genres of esports, inducing a taxonomy that encompasses the variety of these characteristics, and testing findings across domains according to that taxonomy will both expand our methodological tool set for studying high performance teams and refine our theoretical understanding of generalization across domains.

\section{ACKNOWLEDGMENTS}

We would like to thank Professor Melissa Mazmanian and Professor Judy Olson for their help conceptualizing this work.

\section{REFERENCES}

[1] Magnus Bång and Toomas Timpka. 2003. Cognitive tools in medical teamwork: the spatial arrangement of patient records. Methods of information in medicine 42, 4 (2003), 331-336.

[2] Ken R Catchpole, Marc R De Leval, Angus McEwan, Nick Pigott, Martin J Elliott, Annette McQuillan, Carol Macdonald, and Allan J Goldman. 2007. Patient handover from surgery to intensive care: using Formula 1 pit-stop and aviation models to improve safety and quality. Pediatric anesthesia 17, 5 (2007), 470-478.

[3] Simon Cooper and Alan Wakelam. 1999. Leadership of resuscitation teams:'Lighthouse Leadership'. Resuscitation 42, 1 (1999), 27-45.

[4] Lynsey Flowerdew, Ruth Brown, Stephanie Russ, Charles Vincent, and Maria Woloshynowych. 2012. Teams under pressure in the emergency department: an interview study. Emergency Medicine fournal 29, 12 (2012), e2-e2.

[5] Richard M Frankel and Jason J Saleem. 2013. "Attention on the flight deck": what ambulatory care providers can learn from pilots about complex coordinated actions. Patient education and counseling 93, 3 (2013), 367-372.

[6] Diego Gómez-Zará, Leslie A DeChurch, and Noshir S Contractor. 2020. A Taxonomy of Team-Assembly Systems: Understanding How People Use Technologies to Form Teams. Proceedings of the ACM on Human-Computer Interaction 4, CSCW2 (2020), 1-36.

[7] Wayne D Gray. 2017. Game-XP: Action games as experimental paradigms for cognitive science.

[8] J Richard Hackman. 2011. Collaborative intelligence: Using teams to solve hard problems. Berrett-Koehler Publishers.

[9] Edwin Hutchins. 1995. How a cockpit remembers its speeds. Cognitive science 19, 3 (1995), 265-288.

[10] John McCarthy. 1990. Chess as the Drosophila of AI. In Computers, chess, and cognition. Springer, 227-237.

[11] Allen Newell. 1973. You can't play 20 questions with nature and win: Projective comments on the papers of this symposium. (1973).

[12] Jason G Reitman. 2018. Distributed cognition and temporal knowledge in league of legends. International fournal of Gaming and Computer-Mediated Simulations (IFGCMS) 10, 1 (2018), 23-41.

[13] Jason G Reitman, Maria J Anderson-Coto, Minerva Wu, Je Seok Lee, and Constance Steinkuehler. 2020. Esports research: A literature review. Games and Culture 15, 1 (2020), 32-50.

[14] Eduardo Salas, Michael A Rosen, and Heidi King. 2007. Managing teams managing crises: principles of teamwork to improve patient safety in the emergency room and beyond. Theoretical Issues in Ergonomics Science 8, 5 (2007), 381-394.
[15] Jessica L Wildman, Amanda L Thayer, Michael A Rosen, Eduardo Salas, John E Mathieu, and Sara R Rayne. 2012. Task types and team-level attributes: Synthesis of team classification literature. Human Resource Development Review 11, 1 (2012), 97-129.

[16] Daniel Yao, Jason G Reitman, Je Seok Lee, Garrison Wells, and Constance Steinkuehler. 2020. CapturEsports: Data Collection for Teams Research. UCI Esports Conference 2020 . 